\title{
Treatment of ulcers with ablative fractional lasers
}

\author{
Laurel M Morton, MD, ${ }^{1}$ Jeffrey S Dover, MD, ${ }^{1}$ Tania J Phillips, MD,${ }^{1}$ Andrew C Krakowski, MD, ${ }^{2}$ \\ and Nathan S Uebelhoer, $\mathrm{DO}^{3,4^{*}}$
}

\section{Abstract}

Chronic, nonhealing ulcers are a frustrating therapeutic challenge and investigation of innovative therapies continues to be an important research pursuit. One unique and newly applied intervention is the use of ablative fractional lasers. This technology has recently been employed for the treatment of hypertrophic, disfiguring and function-limiting scars, and was first shown to induce healing of chronic wounds in patients with persistent ulcers and erosions within traumatic scars. Recent reports suggest it may be applicable for other types of chronic wounds as well. The mechanism of action for this modality remains to be elucidated but possible factors include laser-induced collagen remodeling, photomicrodebridement and disruption of biofilms, and induction of a proper wound healing cascade.

Semin Cutan Med Surg 34:37-41 @ 2015 Frontline Medical Communications

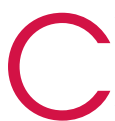
hronic, difficult-to-heal ulcers are a therapeutic challenge, burdensome for patients, and frustrating for clinicians. While the pathophysiology of chronic wounds has been widely studied in order to better understand why some wounds do not heal in a timely fashion, many questions remain as do numerous treatment protocols. Therapies range from basic moisture-retentive dressings and lower extremity compression to new, manufactured biologic dressings and stem-cell therapies. The considerable variety of treatment options points to the fact that many wounds are resistant to typical interventions and therefore, continued ingenuity in the treatment of wounds is essential. One exciting and new intervention is the implementation of ablative fractional (AF) lasers to induce wound healing. Within the past five years, this technology has been applied for the treatment of hypertrophic, disfiguring, and function-limiting scars. While employing AF for this indication, clinicians discovered that it coincidentally demonstrated efficacy in healing persistent trauma-related wounds that exist within scars. ${ }^{1}$ It remains uncertain why this intervention is effective. Proposed mechanisms of action include laser-induced collagen remodeling, microdebridement, disruption of local bio-

\footnotetext{
${ }^{1}$ SkinCare Physicians, Chestnut Hill, Massachusetts.

${ }^{2}$ Rady Children's Hospital, San Diego, California.

${ }^{3}$ Naval Medical Center, San Diego, California.

${ }^{4}$ The Aroostook Medical Center, Presque Isle, Maine.

*Dr Uebelhoer is now with The Aroostook Medical Center.

Disclosures: Drs Morton, Phillips, Krakowski and Uebelhoer have no finan-

cial relationships relevant to this article and no conflict of interests to disclose.

Dr Dover acted as a consultant and has done research for Lumenis.

Correspondence: Laurel M. Morton, MD; SkinCare Physicians, 1244

Boylston Avenue, Chestnut Hill, MA 02467. E-mail: Laurel.m.morton@,

gmail.com,1morton@skincarephysicians.net
}

film, and establishment of a proper wound healing cytokine profile and molecular milieu.

\section{The problem with chronic wounds}

The fact that chronic ulcers account for up to $\$ 25$ billion in health care costs annually in the United States ${ }^{2}$ provides insight to the magnitude of the challenge they present for health care providers. Venous leg ulcers (VLUs), for example, affect between 1\%-1.5\% of the population ${ }^{3}$ and with diabetes and arterial disease, comprise the most common causes of lower extremity wounds, which usually affect the elderly. ${ }^{4}$ Other demographic groups may also be afflicted with difficult-to-heal ulcers. Take for example, the population described by Shumaker and colleagues that includes military veterans who have experienced traumatic blast injuries. These patients are at increased risk of ulceration that is associated with subsequent scarring either from the injury itself or injury-related surgery and skin grafting. ${ }^{1}$

More important than the high health care expense they impose, chronic ulcers are a noteworthy problem because of their significant affect on quality of life. For example, patients with recessive dystrophic epidermolysis bullosa, characterized by an inherited defect in COL7A1, must endure a lifetime of generalized skin fragility, severe blistering, and nonhealing wounds that may be complicated by pain, infection, bleeding, mutilating scarring, and the risk of development of aggressive metastatic squamous cell carcinomas. ${ }^{5}$ In a recent cross-sectional study of 204 patients with chronic venous disease, participants were given a health-related quality of life questionnaire. Those with VLUs demonstrated lower mean scores in 8 quality of life domains including functional capacity, physical aspects, pain, general health status, vitality, social aspects, emotional aspects, and mental health. ${ }^{6}$ Ulcer-related factors that likely contribute to decreased quality of life include, but are not limited to: pain, leakage of exudate and associated odor, bulky bandages, impaired mobility, poor sleep, depression, altered body image, restricted work capacity, and social isolation. ${ }^{7,8} \mathrm{~Pa}-$ tients may also report an elevated sense of powerlessness. ${ }^{9}$ In the case of wounded warriors, scar-associated wounds can complicate the recovery process for those suffering from traumatic blast injuries. For example, in a case presented by Shumaker et al, small erosions and ulcers present at skin graft scars on a lower extremity amputation site prevented progress in the prosthetic rehabilitation of the patient's lower extremity. ${ }^{1}$ Once ulcers have healed, quality of life scores improve, ${ }^{10}$ providing caregivers with a clear incentive to achieve wound healing.

\section{The pathophysiology of chronic wounds}

The causes of chronic wounds are numerous and certainly wound pathophysiology varies based on the ulcer's cause. Yet, there is a robust body of literature that supports some basic tenants of poor 
wound healing that are likely widely applicable. Factors that contribute include poor oxygenation, fibroblast senescence, chronic inflammation, improper function and levels of cytokines and growth factors and their receptors, abnormal matrix metalloproteinase activity and bacterial colonization, and/or infection.

Cellular senescence is meant to protect against tumorigenesis but is problematic in chronic ulcers when fibroblasts are affected. Fibroblasts are essential for dermal matrix proliferation. They lay down structural proteins such as collagen, elastin and extracellular matrix proteins, and produce matrix metalloproteinases (MMPs) that degrade fibrin and facilitate fibroblast migration. ${ }^{11}$ Fibroblasts of chronic VLUs demonstrate a senescent phenotype compared to normal fibroblasts in culture ${ }^{12}$ and do not respond to growth factors like fibroblasts of acute wounds. ${ }^{13}$ Senescent fibroblasts also produce increased proteolytic enzymes such as collagenase, elastase and stromelysin, and decreased tissue inhibitors of metalloproteinases (TIMPs) TIMP-1 and TIMP-3. ${ }^{14}$ These changes promote an environment of degradation rather than proliferation necessary for wound healing.

The normal inflammatory cascade also appears to be disrupted during poor wound healing. Trengrove et al showed that pro-inflammatory interleukin-1 (IL-1), interleukin-6 (IL-6) and tumor necrosis factor- $\alpha$ (TNF- $\alpha$ ) are elevated in the wound fluid of nonhealing ulcers compared to healing ulcers, ${ }^{15}$ however, the difference has not always been reported as statistically significant. ${ }^{16}$ Irregularities in inflammation are also demonstrated by MMPs, which are likely activated by cytokines in chronic wounds. ${ }^{17} \mathrm{Com}-$ pared to wound fluid from acute mastectomy wounds, chronic wound fluid contains drastically elevated levels of MMP-2 and MMP-9;18 and, the MMP-9/TIMP-1 ratio is significantly higher for pressure ulcers that heal poorly compared to those demonstrating successful healing. ${ }^{19}$ Unrestrained protease activity is considered by some to be an underlying factor for nonhealing due to enhanced extracellular matrix turnover. ${ }^{17}$

While wounds are certainly susceptible and can become more difficult to heal due to bacterial infection, it is not clear that a certain bacterial load is associated with impaired healing. ${ }^{20}$ However, it is speculated that bacterial biofilms are relevant factors. Biofilms are microorganism communities which are adherent to a surface or to each other and sequester nutrients, enzymes, and metal ions. In biofilms, bacteria are embedded in a matrix of extracellular polymeric substances consisting of polysaccharides, proteins, glycoproteins, and DNA. ${ }^{20}$ It has been proposed that biofilm-exacerbated oxidative stress contributes to wound chronicity. ${ }^{21}$ Furthermore, the biofilm polysaccharide coating allows bacteria to evade neutrophilic phagocytosis that normally occurs during wound healing. ${ }^{22}$

\section{Ablative fractional laser therapy and its application for scar revision}

Medical laser therapy is understood by the theory of selective photothermolysis, ${ }^{23}$ which describes lasers as energy-emitting devices that release unique wavelengths of light that are absorbed by specific chromophores including hemoglobin, melanin, and water. When a chromophore is exposed to a wavelength in its peak absorption spectra for the appropriate pulse duration, it experiences a rise in temperature and is selectively targeted while surrounding tissue is spared. In the case of AF lasers, made with either carbon dioxide $\left(\mathrm{CO}_{2}\right)$ or erbium: yttrium aluminum garnet (Er:YAG), the targeted chromophore is water. In fractional photothermolysis, introduced by Manstein and colleagues, laser energy is emitted in a grid-like pattern of multiple beams in order to vaporize the stratum corneum and create microscopic columns of coagulation that reach into the epidermis and superficial dermis. ${ }^{24}$ During the subsequent healing process, collagen remodeling is promoted, making this technology a useful tool for ablative facial resurfacing for indications such as dyspigmentation due to photoaging, rhytides, and acne scarring.

Within the last five years, the indications for fractional ablation have expanded to include the treatment of hypertrophic and traumatic scars. In 2009, Waibel and Beer reported the case of a woman with third-degree burn scars to the face, neck, and chest that had already undergone multiple reconstructive surgeries. Following a single session with an $\mathrm{AF} \mathrm{CO}_{2}$ laser, the patient demonstrated a notable improvement of the appearance and texture of the scar. ${ }^{25}$ Subsequently, Cervelli and colleagues published a study of 60 patients with severely scarred skin. Treatment with an $\mathrm{AF} \mathrm{CO}_{2}$ laser resulted in improved skin tone, texture, and appearance in comparison to dermabrasion. ${ }^{26}$

An important step in understanding the broad application of fractional ablation for scarring was initiated by physicians at the Naval Medical Center in San Diego, California. They have been employing this technology for disfiguring and contracture-causing scars related to traumatic blast injuries since $2009 .{ }^{27}$ In 2011 , this group reported the case of a 45 -year-old amputee who experienced multiple traumatic blast injuries while on military deployment. His mobility remained limited due to restricted range of motion despite physical therapy and gait training. On physical exam, his left leg fasciotomy graft site scar was firm and tethered to underlying structures, thereby inhibiting foot movement. This scar was treated with an $\mathrm{AF} \mathrm{CO}_{2}$ laser and after a single session, the patient noticed improved flexibility in the treated leg and less pain with walking. Long-standing gait improvement was maintained after several treatments and measurable passive range of motion was improved at the ankle. ${ }^{28}$ The same group reported a subsequent series of four patients with functional deficits related to scar contracture, all of whom displayed improved range of motion after up to three fractional ablative $\mathrm{CO}_{2}$ treatments. ${ }^{29}$ Laser treatment for scars remains an underutilized tool but certainly has a role aside surgical scar reconstruction. ${ }^{30}$ Similar results have been demonstrated in a pediatric population, as well. In a case series by Krakowski et al, two patients were identified with recalcitrant scar contractures causing persistent functional deficits. After reaching a "plateau" with occupational therapy alone, both patients were treated with ablative fractional laser resurfacing and experienced rapid and cumulative subjective and objective improvements in range of motion and function without reported complications. ${ }^{31}$

\section{Implementation of ablative fractional lasers for chronic wounds}

From the Naval Medical Center in San Diego, Shumaker and colleagues first reported the application of fractional ablation for ulcer healing. They described a 26-year-old serviceman who had five months earlier experienced a traumatic blast injury while in Afghanistan. He had extensive injuries including bilateral above-knee amputations. He was enrolled in a comprehensive rehabilitation program that included wound care and physical and occupational therapy including prosthetic training, functional activity training, 
and gait training. He presented to the dermatology clinic due to skin fragility, sensitivity, and nonhealing areas at the split-thickness skin graft sites at his right amputation stump despite wound care with daily petrolatum or topical bacitracin and intermittent silverimpregnated foam dressings. These erosions and ulcers prevented progress in his prosthetic rehabilitation. In an effort to improve skin texture and pliability, he underwent ablative fractional resurfacing with a $10600 \mathrm{~nm} \mathrm{CO}$ laser (Deep FX laser and UltraPulse Encore system; Lumenis, Ltd, Yokneam, Israel). The entire graft site and $1 \mathrm{~mm}$ to $2 \mathrm{~mm}$ of normal skin was treated with a single pass without overlap at a pulse energy of $50 \mathrm{~mJ}$, treatment density of 5\%, spot size of 120 microns, and pulse width of $250 \mathrm{~ms}$ (the latter two parameters being fixed). Significant wound healing was noted at the patient's first follow up at 1 week post procedure. Continued improvement occurred for two months despite a more aggressive prosthetic rehabilitation regimen. Improved skin texture, pigment and pliability were also noted. Improvement persisted 6 months after his second ablative treatment. The authors reported two other similar cases: a hypertrophic graft site with persistent angulated erosion at a lower extremity amputation stump and a $0.8 \times 1.5 \mathrm{~cm}$ ulcer and scar contracture at the right forearm. Both ulcers healed after fractional ablation. ${ }^{1}$

Several other reports of ulcer healing following AF laser intervention have followed. At the 2014 American Society for Laser Medicine and Surgery (ASLMS) annual meeting in Phoenix, Arizona, Diaz and Krakowski reported the case of an 8-year-old female with hypertrophic burn scars on the dorsal hands following a scalding burn and chemical injury suffered at the age of 15 months. Her left dorsal hand demonstrated a chronic ulcer within a contracture that had failed to respond to typical wound care treatments. Following a single $\mathrm{AF} \mathrm{CO}_{2}$ laser treatment, the chronic ulcer resolved and eventually the patient demonstrated functional and symptomatic improvement. The same group also reported the case of a 17-year-old male with a 6-month history of two nonhealing, noninflammatory ulcers on his left distal shin secondary to liquid nitrogen cryotherapy as treatment for verruca vulgaris. The wounds were treated twice, one month apart, with ablative fractional laser resurfacing using a $\mathrm{CO}_{2}$ laser protocol at a pulse energy of $50 \mathrm{~mJ}$ and treatment density of $5 \%$. The patient achieved complete re-epithelialization of his wounds with only mild postinflammatory hyperpigmentation at the wound sites. ${ }^{32}$

This intervention has been successfully applied to wounds not associated with scarring by these authors and pertinent data was also presented at the 2014 ASLMS meeting. ${ }^{33}$

Most recently, ablative fractional laser resurfacing has demonstrated utility as an adjunct for wound healing in a patient with recessive dystrophic epidermolysis bullosa. Krakowski et al reported the case of a 22-year-old male with recessive dystrophic epidermolysis bullosa who had presented for a painful, nonhealing, 7-cm diameter wound on his left superior back. The wound had been present for nine months and had begun as a small erosion that grew larger with repetitive trauma (eg, sleeping on his back, sitting in a high-back chair, taking off his shirt) despite intensive wound care. The patient was known to be colonized with methicillin-sensitive Staph aureus, Group B Streptococcus, and Pseudomonas aeruginosa, and he had severe allergies to vancomycin and penicillin cross reactors, which limited his ability to receive systemic antibiotics. Using only topical lidocaine $4 \%$ cream under occlusion for an hour, supplemented intraoperatively with ice only, the wound was treated with ablative fractional laser resurfacing using a $\mathrm{CO}_{2}$ laser protocol (pulse energy $30 \mathrm{~mJ}$ and treatment density 5\%). At four weeks postoperatively, the patient's wound measured $2 \mathrm{~cm}$ in diameter, representing a $92 \%$ decrease in wound surface area. He was treated a second time, and at his two-week follow-up he was noted to have near-complete re-epithelialization of the wound. Additionally, the patient reported being "pain free" and was less demoralized. $^{34}$

This treatment modality for wound care remains in its infancy and even experts may struggle with choosing the appropriate patients, devices, and specific treatment settings. While ulcer treatments reported to date have utilized $\mathrm{CO}_{2}$-based devices, fractional Er:YAG devices should theoretically also be effective. $\mathrm{CO}_{2}$ lasers possess a greater potential for surrounding tissue coagulation and likely decreased procedure-associated bleeding, which is a relevant consideration for larger open wounds. ${ }^{30}$

A 2014 consensus statement on laser treatment of traumatic scars proposed treatment parameters that may be modified and applied in the treatment of wounds as well. The consensus panel suggested that an important factor for guiding treatment settings is an estimation of scar thickness. Treatment depth, which correlates with a selected energy setting, should be proportional to but not greater than scar thickness. Furthermore, providers should minimize concurrent therapies and apply low-treatment densities, narrow-beam diameters and short-pulse widths. The goal is to decrease risk of scar worsening. Generally, only one pass per treatment is recommended with a goal for a series of treatments at 2-3 month intervals until the desired endpoint is reached (often 3-6 treatments total). ${ }^{30}$ When applying these principles to the treatment of wounds, it is important to consider that open wounds do not have the protective covering of stratum corneum or epithelium and therefore, decreased energy settings may be more appropriate. The wound edges should also be treated for a width ranging from $2 \mathrm{~mm}-2 \mathrm{~cm}$. Following treatment, adequate moisture-retentive wound dressings should be applied as well as any indicated adjuvant measures such as compression therapy. These authors have experienced that patients do well with topical anesthesia alone but this must be applied judiciously as systemic absorption may be more likely to occur through an open ulcer rather than stratum corneum-protected normal skin.

\section{Proposed mechanisms of action}

It is interesting to postulate why fractional ablation improves wound healing. Contributing factors may include improved scar texture and pliability that lead to decreased tension, a possible contributing factor in nonhealing scar-related wounds. Healing occurring in ulcers not related to scarring suggests that at least one mechanism of action of this technique does not depend on decreasing scar-related tension and contracture. The prospect that tiny columns of coagulation caused by AF lasers contribute to tissue debridement and/or biofilm disruption may also be relevant. Finally, it is well documented that fractional ablation changes the molecular profile of skin and perhaps this creates an environment more amenable to proper wound healing.

Prior to the availability of lasers, scar reconstruction was limited in large part to surgical intervention. The presence of ulcers within hypertrophic scars, such as burn scars, is a well-known phenom- 
enon and has largely been attributed to the tension existing within such scars and resulting skin fragility. Tension leads to tissue ischemia, which can negatively influence epidermal stability. Therefore, surgical intervention aimed at relieving tension can lead to ulcer improvement as well. Potential procedures include release and grafting and Z-plasties. ${ }^{35}$

It is possible that the laser releases scar tension because it induces microscopic collagen remodeling. Normal skin collagen is present as fine fibrils in a basket weave pattern, but scar tissue shows thick parallel bundles of cellular collagen. ${ }^{36}$ It has been established with histologic evidence, on multiple occasions, that an AF laser induces long-standing changes in collagen structure as well as new collagen formation, or neocollagenesis.

In El-Domyati et al's study of 12 patients treated with either a fractional or fully ablative Er:YAG laser, skin biopsies for histologic evaluation were taken prior to treatment and at 1 and 6 months post treatment. Immunohistochemistry showed increased collagen I and III concentrations in both treatment groups at 1 and 6 months (without a statistically significant difference in concentrations between groups) as well as thinner, newer collagen bundles supporting laser-induced collagen remodeling. ${ }^{37}$ In 2014, Makboul et al demonstrated collagen remodeling following fractional $\mathrm{CO}_{2}$ treatment of hypertrophic scars as well with histologic evidence showing the replacement of irregular collagen bands with organized new collagen fibrils. ${ }^{38}$ Confocal microscopy has also demonstrated this transition of collagen to long, straight fibers in parallel alignment following fractional $\mathrm{CO}_{2}$ ablation. ${ }^{39}$ Clinically speaking, these microscopic changes in collagen result in improved skin texture, pliability, and function. ${ }^{1}$

Furthermore, multiple studies have reported molecular changes that support collagen remodeling. In a 2007 study, Hantash and colleagues established that heat shock protein (HSP) 47 is detected at 7 days post fractional ablative treatment and remains elevated for at least three months. ${ }^{40}$ HSP 47 is important for wound healing in that it promotes neocollagenesis. ${ }^{41}$ Subsequent studies support the upregulation of this protein. ${ }^{42,43} \mathrm{~A}$ fractional $\mathrm{CO}_{2}$ laser also induces upregulated gene expression of select MMPs, which are important for the denaturation, degradation and reorganization of collagen allowing for new, well-organized collagen bundles. ${ }^{44}$ This is true following fractional $\mathrm{CO}_{2}$ treatment of burn scars as well. ${ }^{45}$

Typical care protocols for chronic wounds often include debridement of devitalized tissue whether by surgical, autolytic, mechanical, enzymatic, or biologic means. ${ }^{46}$ Eschars may serve as proinflammatory stimuli that inhibit normal healing and slough can serve as a medium for bacterial growth. ${ }^{46}$ It is likely that mechanical debridement can be effective at decreasing bacterial load and possibly biofilms that inhibit healing and contribute to wound chronicity. ${ }^{46-48}$ It has been proposed by Shumaker and colleagues that fractional ablation may enact photomicrodebridement, disrupting biofilms which are otherwise resistant to antibiotics. ${ }^{1}$ Fractional laser intervention may also decrease the burden of devitalized tissue and senescent wound fibroblasts because of its ablative nature.

Finally, we know that AF lasers change the molecular milieu in treated tissue. In addition to the upregulation of HSPs and MMPs, multiple studies have supported the idea that fractional ablation initiates other aspects of a proper wound healing cascade likely due to thermal or photothermolytic ${ }^{49}$ tissue injury. For instance,
Prigano et al treated 18 patients with photodamaged facial skin with fractional ablative $\mathrm{CO}_{2}$ lasers and demonstrated elevated levels of wound healing molecules including platelet-derived growth factor (PDGF) and endothelial growth factor (EGF) by immunohistochemistry. ${ }^{50} \mathrm{PDGF}$ is chemotactic for important inflammatory cells in early wound healing such as macrophages and neutrophils and also stimulates fibroblasts to create extracellular matrix. ${ }^{50}$ EGF is important for re-epithelialization. ${ }^{50}$ Jiang and colleagues have used a mouse model to further elucidate alterations in cytokine profile following fractional laser treatments. They showed an increase in vascular endothelial growth factor (VEGF) at 1 and 3 days post laser treatment in endothelial cells and keratinocytes. ${ }^{51}$ VEGF is important for wound healing through multiple means including collagen deposition, angiogenesis, macrophage recruitment and epithelialization..$^{51,52}$

Jiang's group also utilized a mouse model to demonstrate elevated levels of transforming growth factor $\beta 1$ (TGF- $\beta 1$ ) at day 1 and 7 post fractional $\mathrm{CO}_{2}$ laser treatment with normalization of levels by day $14 .{ }^{53}$ TGF- $\beta 1$ is critical for wound healing and produced by fibroblasts, macrophages and keratinocytes. It plays a role in inflammatory cell recruitment, angiogenesis, re-epithelialization, and connective tissue reconstruction during wound healing. ${ }^{53}$ When over-expressed, TGF- $\beta 1$ can lead to scarring but in the mouse study, levels of this cytokine began to decrease again one day after fractional treatment. Interestingly, a 2014 study including 8 patients with hypertrophic scarring treated with a fractional $\mathrm{CO}_{2}$ laser showed that TGF- $\beta 1$ levels are actually decreased within hypertrophic scar tissue six months after treatment ${ }^{38}$ suggesting that fractional treatment may regulate TGF- $\beta 1$ to more appropriate levels. Thus, in addition to promoting wound healing, AF laser's ability to decrease the severity of scar tissue may also enhance wound healing in scar-related wounds.

\section{Conclusions}

The treatment of difficult-to-heal wounds can be exasperating for both physicians and patients, and new and effective treatment regimens are highly sought after. Though the use of fractional ablation for chronic wounds is novel, there are sufficient reported examples to suggest it is an efficacious intervention for at least certain patients. To best elucidate appropriate treatment protocols, it will be important to conduct larger, randomized and controlled trials. Additionally, a better understanding of its mechanism of action may also help providers choose patients that are most likely to respond to this treatment intervention.

\section{References}

1. Shumaker PR, Kwan JM, Badiavas EV, Waibel J, Davis S, Uebelhoer NS. Rapid healing of scar-associated chronic wounds after ablative fractional resurfacing. Arch Dermatol, 2012;148(11):1289-1293.

2. Sen CK, Gordillo GM, Roy S, et al. Human skin wounds: a major and snowballing threat to public health and the economy. Wound Repair Regen. 2009;17(6):763771.

3. van Gent WB, Wilschut ED, Wittens C. Management of venous ulcer disease. BMJ. 2010;341:c6045.

4. Callam MJ, Ruckley CV, Harper DR, Dale JJ. Chronic ulceration of the leg: extent of the problem and provision of care. BrMed J (Clin Res Ed). 1985;290(6485):18551856.

5. Fine JD, Johnson LB, Weiner M, Li KP, Suchindran C. Epidermolysis bullosa and the risk of life-threatening cancers: the National EB Registry experience, 19862006. J Am Acad Dermatol. 2009;60(2):203-211.

6. Dias TY, Costa IK, Melo MD, Torres SM, Maia EM, Torres Gde V. Quality of life 
assessment of patients with and without venous ulcer [in English, Portuguese, Spanish]. Rev Lat Am Enfermagem. 2014;22(4):576-581.

7. Hopman WM, VanDenKerkhof EG, Carley ME, Kuhnke JL, Harrison MB. Factors associated with health-related quality of life in chronic leg ulceration. Qual Life Res. 2014;23(6):1833-1840.

8. Maddox D. Effects of venous leg ulceration on patients' quality of life. Nurs Stand. 2012;26(38):42-49.

9. de Almeida SA, Salomé GM, Dutra RA, Ferreira LM. Feelings of powerlessness in individuals with either venous or diabetic foot ulcers. J Tissue Viability. 2014;23(3):109-114.

10. Iglesias CP, Birks Y, Nelson EA, Scanlon E, Cullum NA. Quality of life of people with venous leg ulcers: a comparison of the discriminative and responsive characteristics of two generic and a disease specific instruments. Qual Life Res. 2005;14(7):1705-1718.

11. Bainbridge P. Wound healing and the role of fibroblasts. $J$ Wound Care. 2013;22(8):407-408, 410-412.

12. Mendez MV, Stanley A, Park HY, Shon K, Phillips T, Menzoian JO. Fibroblasts cultured from venous ulcers display cellular characteristics of senescence. J Vasc Surg. 1998;28(5):876-883.

13. Shah JM, Omar E, Pai DR, Sood S. Cellular events and biomarkers of wound healing. Indian J Plast Surg. 2012;45(2):220-228.

14. Harding KG, Moore K, Phillips TJ. Wound chronicity and fibroblast senescence-implications for treatment. Int Wound J. 2005;2(4):364-368.

15. Trengove NJ, Bielefeldt-Ohmann H, Stacey MC. Mitogenic activity and cytokine levels in non-healing and healing chronic leg ulcers. Wound Repair Regen. 2000;8(1):13-25.

16. Harris IR, Yee KC, Walters CE, et al. Cytokine and protease levels in healing and non-healing chronic venous leg ulcers. Exp Dermatol. 1995;4(6):342-349.

17. Raffetto, JD. Inflammation in chronic venous ulcers. Phlebology. 2013;28 Suppl 1:61-67.

18. Wysocki AB, Staiano-Coico L, Grinnell F. Wound fluid from chronic leg ulcers contains elevated levels of metalloproteinases MMP-2 and MMP-9. J Invest Dermatol. 1993;101(1):64-68.

19. Ladwig GP, Robson MC, Liu R, Kuhn MA, Muir DF, Schultz GS. Ratios of activated matrix metalloproteinase- 9 to tissue inhibitor of matrix metalloproteinase- 1 in wound fluids are inversely correlated with healing of pressure ulcers. Wound Repair Regen. 2002;10(1):26-37.

20. Percival SL, Thomas JG, Williams DW. Biofilms and bacterial imbalances in chronic wounds: anti-Koch. Int Wound J. 2010;7(3):169-175.

21. Dhall S, Do D, Garcia M, et al. A novel model of chronic wounds: importance of redox imbalance and biofilm-forming bacteria for establishment of chronicity. PLoS One. 2014;9(10):e109848.

22. Scali C, Kunimoto B. An update on chronic wounds and the role of biofilms. J Cutan Med Surg, 2013;17(6):371-376.

23. Anderson RR, Parrish JA. Selective photothermolysis: precise microsurgery by selective absorption of pulsed radiation. Science. 1983;220(4596):524-527.

24. Manstein D, Herron GS, Sink RK, Tanner H, Anderson RR. Fractional photothermolysis: a new concept for cutaneous remodeling using microscopic patterns of thermal injury. Lasers Surg Med. 2004;34(5):426-438.

25. Waibel J, Beer K. Ablative fractional laser resurfacing for the treatment of a thirddegree burn. J Drugs Dermatol, 2009;8(3):294-297.

26. Cervelli V, Gentile P, Spallone D, et al. Ultrapulsed fractional $\mathrm{CO}_{2}$ laser for the treatment of post-traumatic and pathological scars. J Drugs Dermatol. 2010;9(11):13281331.

27. Uebelhoer NS, Ross EV, Shumaker PR. Ablative fractional resurfacing for the treatment of traumatic scars and contractures. Semin Cutan Med Surg. 2012;31(2):110120.

28. Kwan JM, Wyatt M, Uebelhoer NS, Pyo J, Shumaker PR. Functional improvement after ablative fractional laser treatment of a scar contracture. PM R. 2011;3(10):986-987.

29. Shumaker PR, Kwan JM, Landers JT, Uebelhoer NS. Functional improvements in traumatic scars and scar contractures using an ablative fractional laser protocol. $J$ Trauma Acute Care Surg. 2012;73(2 Suppl 1):S116-S121.

30. Anderson RR, Donelan MB, Hivnor C, et al. Laser treatment of traumatic scars with an emphasis on ablative fractional laser resurfacing: consensus report. JAMA Dermatol. 2014;150(2):187-193.

31. Krakowski AC, Goldenberg A, Eichenfield LF, Peck-Murray J, Shumaker PR. Ablative fractional laser resurfacing helps treat restrictive pediatric scar contractures. Pediatrics. 2014;134(6):e1700-E1705.

32. Diaz L, Krakowski A. Rapid Healing of a Chronic Ulcer Within a Burn Scar in a Pediatric Patient Following a Single Ablative Fractional Resurfacing Treatment. Paper presented at: $34^{\text {th }}$ American Society for Laser Medicine \& Surgery Annual International Conference; April 4-6, 2014; Phoenix, AZ

33. Morton L, Phillips T, Uebelhoer N, Dover J. New and Innovative Use of Ablative Fractional $\mathrm{CO}_{2}$ Laser in Treatment of Post Traumatic Chronic Lower Extremity Ulcers. Paper presented at: $34^{\text {th }}$ American Society for Laser Medicine \& Surgery Annual International Conference; April 4-6, 2014; Phoenix, AZ.

34. Krakowski AC, Ghasri P. Case report: rapidly healing epidermolysis bullosa wound after ablative fractional resurfacing. Pediatrics. 2015;135(1)e207-e210.

35. Donelan, MB. Principles of Burn Reconstruction. In: Thorne $\mathrm{CH}$, ed. Grabb and Smith's Plastic Surgery. $6^{\text {th }}$ ed. Philadelphia, PA: Lippincott Williams \& Wilkins; 2007.

36. Weedon D. Skin Pathology. 2nd ed. New York, NY: Churchill Livingstone; 2002

37. El-Domyati M, Abd-El-Raheem T, Abdel-Wahab H, et al. Fractional versus ablative erbium:yttrium-aluminum-garnet laser resurfacing for facial rejuvenation: an objective evaluation. J Am Acad Dermatol. 2013;68(1):103-112.

38. Makboul M, Makboul R, Abdelhafez AH, Hassan SS, Youssif SM. Evaluation of the effect of fractional $\mathrm{CO}_{2}$ laser on histopathological picture and TGF- 31 expression in hypertrophic scar. J Cosmet Dermatol. 2014;13(3):169-179.

39. Longo C, Falimberti M, De Pace B, Pellacani G, Bencini PL. Laser skin rejuvenation: epidermal changes and collagen remodeling evaluated by in vivo confocal microscopy. Lasers Med Sci. 2013;28(3):769-776.

40. Hantash BM, Bedi VP, Kapadia B, et al. In vivo histological evaluation of a novel ablative fractional resurfacing device. Lasers Surg Med. 2007;39(2):96-107.

41. Keagle JN, Welch WJ, Young DM. Expression of heat shock proteins in a linear rodent wound. Wound Repair Regen. 2001;9(5):378-385.

42. Helbig D, Bodendorf MO, Grunewald S, Kendler M, Simon JC, Paasch U. Immunohistochemical investigation of wound healing in response to fractional photothermolysis. J Biomed Opt. 2009;14(6):064044.

43. Xu XG, Luo YJ, Wu Y, et al. Immunohistological evaluation of skin responses after treatment using a fractional ultrapulse carbon dioxide laser on back skin. Dermatol Surg. 2011;37(8):1141-1149.

44. Reilly MJ, Cohen M, Hokugo A, Keller GS. Molecular effects of fractional carbon dioxide laser resurfacing on photodamaged human skin. Arch Facial Plast Surg. 2010;12(5):321-325

45. Qu L, Liu A, Zhou L, et al. Clinical and molecular effects on mature burn scars after treatment with a fractional CO(2) laser. Lasers Surg Med. 2012;44(7):517-524

46. Gary Sibbald R, Goodman P, Reneeka P. Wound bed preparation 2012. J Cutan Med Surg. 2013;17 Suppl 1:S12-S22.

47. Hurlow J, Bowler PG. Clinical experience with wound biofilm and management: case series. Ostomy Wound Manage. 2009;55(4):38-49.

48. Wilkins RG, Unverdorben M. Wound cleaning and wound healing: a concise review. Adv Skin Wound Care. 2013;26(4):160-163.

49. Helbig D, Paasch U. Molecular changes during skin aging and wound healing after fractional ablative photothermolysis. Skin Res Technol. 2011;17(1):119-128.

50. Prignano F, Campolmi P, Bonan P, et al. Fractional $\mathrm{CO}_{2}$ laser: a novel therapeutic device upon photobiomodulation of tissue remodeling and cytokine pathway of tissue repair. Dermatol Ther. 2009;22 Suppl 1:S8-S15.

51. Jiang, X, Ge H, Zhou C, Chai X, Ren QS. The role of vascular endothelial growth factor in fractional laser resurfacing with the carbon dioxide laser. Lasers Med Sci. 2012;27(3):599-606.

52. Johnson KE, Wilgus TA. Vascular Endothelial Growth Factor and Angiogenesis in the Regulation of Cutaneous Wound Repair. Adv Wound Care (New Rochelle). 2014;3(10):647-661.

53. Jiang $\mathrm{X}$, Ge $\mathrm{H}$, Zhou $\mathrm{C}$, Chai $\mathrm{X}$, Deng $\mathrm{H}$. The role of transforming growth factor $B 1$ in fractional laser resurfacing with a carbon dioxide laser. Lasers Med Sci. 2014:29(2):681-687. 